\title{
Partial relay-based cooperative primary user detection in cognitive radio networks
}

\author{
Shengliang Peng ${ }^{1,2^{*}}, \mathrm{Xi}_{\mathrm{Y}} \mathrm{Yang}^{3}$, Shuli Shu ${ }^{1}$ and Xiuying $\mathrm{CaO}^{3}$
}

\begin{abstract}
In order to identify spectrum holes, secondary users (SUs) should detect whether the spectrum band is occupied by primary user (PU). Cooperative PU detection is the most prevalent PU detection method in cognitive radio (CR) networks. Existing cooperative PU detection usually consumes a dedicated reporting channel to share the detection results. This paper proposes a partial relay-based cooperative PU detection scheme that does not require any reporting channels. Our idea is to let the SU with higher signal-to-noise ratio (SNR) sacrifices part of its detection period acting as the relay node to help other SUs. Although the performance of this SU is inevitably impaired, other SUs may benefit from the relay, and performance of the whole CR network can be improved. Moreover, this paper investigates the relay policy of proposed scheme under two criteria. Considering the criterion of balancing detection accuracy, an interesting conclusion that the optimal relay policy merely depends on the SNR gap is derived. Both lower and upper bounds of the optimal relay policy are deduced as well. Considering the criterion of maximizing detection agility, numerical approach to obtain the optimal relay policy is introduced, and the agility gain is also analyzed. Simulation results are provided to verify all conclusions above.
\end{abstract}

Keywords: Partial relay, Cooperative PU detection, Relay policy, Detection accuracy, Detection agility

\section{Introduction}

The dramatic flourish of wireless technologies has aroused great concern about spectrum resources in recent years [1-3]. Coined by Mitola [4], cognitive radio (CR) is regarded as a promising solution to the so-called "spectrum scarcity" issue by enabling the users without dedicated spectrum resources, namely secondary users (SUs), to reuse the temporal and spatial "holes" on the spectrum band that has been licensed to the primary user (PU) for exclusive use. In order to identify spectrum holes, SUs should detect whether PU is occupying its band $[5,6]$.

Various methods have been suggested for PU detection, and cooperative PU detection increasingly becomes the most prevalent choice as it exploits the diversity of multiple SUs in CR networks [7]. Based on how multiple SUs share their detection results, cooperative PU detection can be classified into three categories [8]: centralized

*Correspondence: peng.shengliang@gmail.com

${ }^{1}$ College of Information Science and Engineering, Huaqiao University, 668 Jimei Avenue, 361021 Xiamen, China

${ }^{2}$ Department of Electrical and Computer Engineering, Stevens Institute of Technology, Castle Point, Hoboken, 07030 USA

Full list of author information is available at the end of the article manner that all SUs forward the results to a fusion center (FC) $[7,9-11]$, distributed manner that SUs communicate with others directly [12], and relay-assisted manner that one or more SUs serve as relays to assist other SUs in forwarding the results $[13,14]$. No matter in which category of cooperation, in order to avoid interfering with PU, a dedicated reporting channel (or control channel) is used to transmit detection results, not only consuming extra spectrum resource but also incurring additional transmission errors as the channel cannot be perfect [15]. In [16], a selective-relay-based scheme is proposed to upload SUs' binary detection results to FC on the licensed band without causing severe interference to PU. However, after combining SUs' detection results, FC should feed its global decision back to SUs and inform them of either using or vacating the licensed band. This feedback link (from FC to SUs) still needs extra channel resources.

This paper concentrates on cooperative PU detection and proposes a partial relay-based scheme that does not require any dedicated channels. To achieve this goal, $\langle 1\rangle$ PU signal that can be transmitted on licensed band is relayed instead of SUs' detection results, and $\langle 2\rangle$ decisions are individually made by SUs themselves to avoid 
feeding back the global decision. Different from existing partial relay selection techniques [17], the term "partial relay" in our scheme refers to that one SU spends part of its detection period acting as the relay node. Although the performance of relaying SU is inevitably impaired as its detection time is reduced, other SUs in the CR network may benefit from the relay mechanism, and thus, our scheme could be superior to the non-relay scheme from the entire CR network point of view, e.g., via improving the network detection agility. Moreover, this paper investigates how much of the detection period should be used for partial relay and deduces the optimal relay policy under the criteria of balancing detection accuracy and maximizing detection agility.

The rest of this paper is organized as follows. Section 2 introduces the model of partial relay. Section 3 analyzes the performance of partial relay-based cooperative $\mathrm{PU}$ detection. In Section 4, the impact of relay policy is studied under different criteria. Section 5 provides some simulation results, and Section 6 concludes this paper.

\section{Partial relay model}

Similar to [13, 18], a CR network with two SUs, namely $S U_{1}$ and $S U_{2}$, is considered for simplicity, as shown in Fig. 1. If there exist more than two SUs in the CR network, similar relay mechanism can be conducted by choosing one $\mathrm{SU}$ with the highest signal-to-noise ratio (SNR) as the relay according to literature [19]. Due to lack of spectrum resource, SUs periodically detect whether the licensed band is being used by PU; if a spectrum hole is found, they use different sub-channels of the band to transmit.

In case of non-cooperation, each SU individually detects PU during the detection period $T_{d}$; if the absence of PU is detected, they transmit on the licensed band during the period $T_{c}$, as depicted in Fig. 2a.

Because of various propagation conditions, the received signal-to-noise ratios (SNRs) of $S U_{1}$ and $S U_{2}$, denoted by $\gamma_{1}$ and $\gamma_{2}$, respectively, are usually different. Therefore, given the same detection period $T_{d}$, the SU with higher

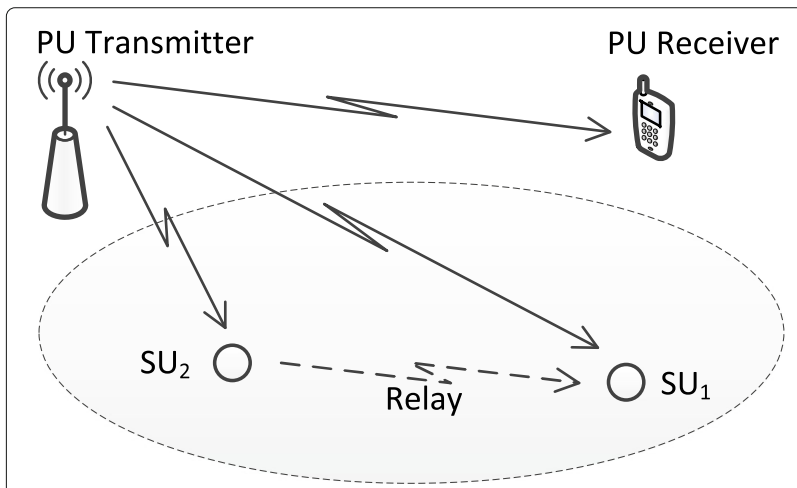

Fig. 1 Two-SU CR network

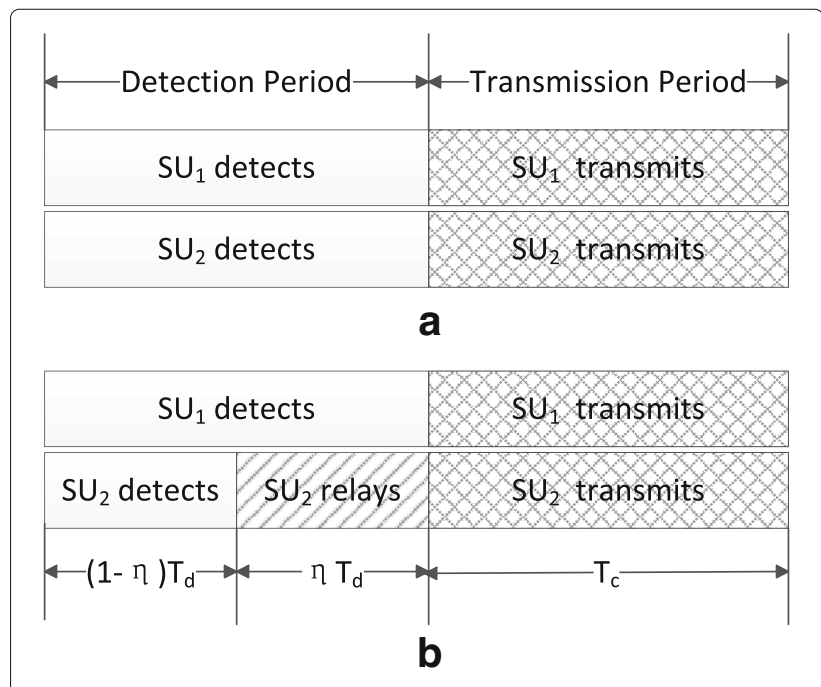

Fig. 2 a Non-cooperation, and b partial relay-based cooperation

SNR (say $S U_{2}$ ) may perform detection with excessive accuracy, while the SU with lower SNR (say $S U_{1}$ ) may suffer from numerous missed detections and cause intolerable interference to PU. Our idea is to let $S U_{2}$ sacrifice part of its detection period $\eta T_{d}(0<\eta<1)$ to help $S U_{1}$ via relay. Then, the whole detection period of $S U_{2}$ is divided into two phases, as depicted in Fig. 2b.

In the first phase of $(1-\eta) T_{d}$, both $S U_{1}$ and $S U_{2}$ perform PU detection as before. The received signals of $S U_{1}$ and $\mathrm{SU}_{2}$ are given by

$$
\begin{gathered}
y_{1}^{(1)}=h_{1}(k) \cdot s(k)+n_{1}(k), \\
y_{2}(k)=h_{2}(k) \cdot s(k)+n_{2}(k),
\end{gathered}
$$

where $s(k)$ denotes the signal of PU; $s(k)=0$ if $\mathrm{PU}$ is absent; $h_{1}(k)$ and $h_{2}(k)$ denote the channels from PU transmitter to $S U_{1}$ and $S U_{2}$, respectively; $n_{1}(k)$ and $n_{2}(k)$ denote the additive noises of $S U_{1}$ and $S U_{2}$, respectively.

In the second phase of $\eta T_{d}, S U_{2}$ stops detecting and begins to relay its received signal in an immediate amplifyand-forward manner. Note that the signal is forwarded on licensed band, and thus, no extra channel is required. Although this signal affects PU receiver simultaneously, it can be treated as a multipath copy of original PU signal and collected by use of Rake technique. Consequently, the received signal of $S U_{1}$ in this phase can be expressed as

$$
\begin{aligned}
y_{1}^{(2)}(k)= & h_{1}(k) \cdot s+\sqrt{\kappa} h_{21}(k) \cdot y_{2}(k)+n_{1}(k) \\
= & {\left[h_{1}(k)+\sqrt{\kappa} h_{21}(k) h_{2}(k)\right] s(k) } \\
& +\left[n_{1}(k)+\sqrt{\kappa} h_{21}(k) n_{2}(k)\right],
\end{aligned}
$$

where $\kappa$ denotes the relay gain, $h_{21}(k)$ denotes the channel from $S U_{2}$ to $S U_{1}$.

Consider $s(k)$ is binary phase shift keying signal; $h_{1}(k), h_{2}(k)$, and $h_{21}(k)$ are real Gaussian with zero means; 
$n_{1}(k)$ and $n_{2}(k)$ are zero mean and unit variance Gaussian white noises; all these random processes are independent of each other [20]. Then, the SNR of $S U_{1}$ in the second phase is

$$
\begin{aligned}
\gamma_{1}^{(2)} & =\frac{E\left\{\left|\left[h_{1}(k)+\sqrt{\kappa} h_{21}(k) h_{2}(k)\right] s(k)\right|^{2}\right\}}{E\left\{\left|n_{1}(k)+\sqrt{\kappa} h_{21}(k) n_{2}(k)\right|^{2}\right\}} \\
& =\frac{\gamma_{1}+\kappa \gamma_{h} \gamma_{2}}{1+\kappa \gamma_{h}},
\end{aligned}
$$

where $\gamma_{h}$ denotes the impact of the relay channel from $S_{2}$ to $S U_{1}$,

$$
\begin{aligned}
& \gamma_{h}=E\left\{\left|h_{21}(k)\right|^{2}\right\}, \\
& \gamma_{1}=\frac{E\left\{\left|h_{1}(k) s(k)\right|^{2}\right\}}{E\left\{\left|n_{1}(k)\right|^{2}\right\}}, \\
& \gamma_{2}=\frac{E\left\{\left|h_{2}(k) s(k)\right|^{2}\right\}}{E\left\{\left|n_{2}(k)\right|^{2}\right\}} .
\end{aligned}
$$

Since $\gamma_{2}>\gamma_{1}$, it is obvious that $\gamma_{1}^{(2)}>\gamma_{1}$ according to (4). In other words, the SNR of $S U_{1}$ is improved via relay, and the benefit of partial relay is proved.

\section{Performance analysis}

Without loss of generality, assume both SUs adopt energy detector to make decisions throughout this paper $[7,9,10,20]$. The observed energy of $S U_{1}$ and $S U_{2}$ can be calculated as

$$
\begin{aligned}
v_{1} & =v_{1}^{(1)}+v_{1}^{(2)} \\
& =\sum_{k=1}^{n_{1}}\left|y_{1}^{(1)}(k)\right|^{2}+\sum_{k=n_{1}+1}^{n}\left|y_{1}^{(2)}(k)\right|^{2}, \\
v_{2} & =\sum_{k=1}^{n_{1}}\left|y_{2}(k)\right|^{2},
\end{aligned}
$$

where $v_{1}^{(1)}$ and $v_{1}^{(2)}$ are the energy observed by $S U_{1}$ in the first and the second phases, respectively; $n=T_{d} f_{s}$ and $n_{1}=(1-\eta) T_{d} f_{s}$ are the sample numbers of the whole detection period and its first phase, respectively; $f_{s}$ is the sampling frequency.

Comparing $v_{1}$ and $v_{2}$ with corresponding thresholds, $S U_{1}$ and $S U_{2}$ can make decisions individually by themselves rather than obtain the global decision from FC via a dedicated channel,

$$
\left\{\begin{array}{l}
v_{i} \leq \lambda_{i} S U_{i} \text { accpect } H_{0} \\
v_{i}>\lambda_{i} S U_{i} \text { accpect } H_{1}
\end{array}, i=1,2,\right.
$$

where $\lambda_{1}$ and $\lambda_{2}$ represent the decision thresholds of $S U_{1}$ and $S U_{2}$, respectively; $H_{0}$ and $H_{1}$ represent the hypotheses of PU's absence and presence, respectively.

Since the sample number used by energy detector is usually very large, $v_{1}^{(1)}, v_{1}^{(2)}$, and $v_{2}$ approximately follow Gaussian distributions according to the central limit theorem.
The means and variances of these Gaussian variables can be derived as follows $[18,20]$,

$$
\begin{aligned}
& E\left\{v_{1}^{(1)}\right\}= \begin{cases}n(1-\eta) & H_{0}, \\
n(1-\eta)\left(1+\gamma_{1}\right) & H_{1}\end{cases} \\
& \operatorname{Var}\left\{v_{1}^{(1)}\right\}= \begin{cases}2 n(1-\eta) & H_{0}, \\
2 n(1-\eta)\left(1+\gamma_{1}\right)^{2} H_{1}\end{cases} \\
& E\left\{v_{1}^{(2)}\right\}=\left\{\begin{array}{ll}
n \eta\left(1+\kappa \gamma_{h}\right) & H_{0} \\
n \eta\left(1+\gamma_{1}+\kappa \gamma_{h}+\kappa \gamma_{h} \gamma_{2}\right) & H_{1}
\end{array},\right.
\end{aligned}
$$

$$
\operatorname{Var}\left\{v_{1}^{(2)}\right\}=\left\{\begin{array}{cc}
2 n \eta\left[\left(1+\kappa \gamma_{h}\right)^{2}+3 \kappa^{2} \gamma_{h}^{2}\right] & H_{0} \\
2 n \eta\left[\left(1+\gamma_{1}+\kappa \gamma_{h}+\kappa \gamma_{h} \gamma_{2}\right)^{2}\right. & \\
\left.+3 \kappa^{2} \gamma_{h}^{2}\left(1+\gamma_{2}\right)^{2}\right] & H_{1}
\end{array},\right.
$$

$$
\begin{gathered}
E\left\{v_{2}\right\}= \begin{cases}m_{20}=n(1-\eta) & H_{0}, \\
m_{21}=n(1-\eta)\left(1+\gamma_{2}\right) & H_{1}\end{cases} \\
\operatorname{Var}\left\{v_{2}\right\}=\left\{\begin{array}{l}
\delta_{20}^{2}=2 n(1-\eta) \\
\delta_{21}^{2}=2 n(1-\eta)\left(1+\gamma_{2}\right)^{2}
\end{array} H_{1}\right.
\end{gathered} .
$$

Note that $v_{1}^{(1)}$ is independent of $v_{1}^{(2)}$, and thus $v_{1}=v_{1}^{(1)}+$ $v_{1}^{(2)}$ also follows Gaussian distribution with the mean and variance as below,

$$
\begin{aligned}
E\left\{v_{1}\right\} & =E\left\{v_{1}^{(1)}+v_{1}^{(2)}\right\} \\
& =\left\{\begin{array}{ll}
m_{10}=n\left(1+\eta \kappa \gamma_{h}\right) & H_{0} \\
m_{11}=n\left(1+\gamma_{1}+\eta \kappa \gamma_{h}+\eta \kappa \gamma_{h} \gamma_{2}\right) & H_{1}
\end{array},\right.
\end{aligned}
$$

$$
\begin{aligned}
\operatorname{Var}\left\{v_{1}\right\} & =\operatorname{Var}\left\{v_{1}^{(1)}+v_{1}^{(2)}\right\} \\
& =\left\{\begin{array}{cc}
\delta_{10}^{2}=2 n\left(1+2 \eta \kappa \gamma_{h}+4 \eta \kappa^{2} \gamma_{h}^{2}\right) & H_{0} \\
\delta_{11}^{2}=2 n\left[\left(1+\gamma_{1}\right)^{2}+2 \eta \kappa \gamma_{h}\left(1+\gamma_{2}\right)\right. \\
\left.\cdot\left(1+\gamma_{1}+2 \kappa \gamma_{h}+2 \kappa \gamma_{h} \gamma_{2}\right)\right]
\end{array} H_{1}\right.
\end{aligned} .
$$

For simplicity, we assume that the relay gain is properly chosen to compensate for the path loss of relay channel, that is, $\kappa=1 / \gamma_{h}$. In this case, the mean and variance of $v_{1}$ can be rewritten as

$$
\begin{aligned}
& E\left\{v_{1}\right\}= \begin{cases}m_{10}=n(1+\eta) & H_{0}, \\
m_{11}=n\left(1+\gamma_{1}+\eta+\eta \gamma_{2}\right) & H_{1}\end{cases} \\
& \operatorname{Var}\left\{v_{1}\right\}=\left\{\begin{array}{c}
\delta_{10}^{2}=2 n(1+6 \eta) \\
\delta_{11}^{2}=2 n\left[\left(1+\gamma_{1}\right)^{2}+2 \eta\right. \\
\left.\cdot\left(1+\gamma_{2}\right)\left(3+\gamma_{1}+2 \gamma_{2}\right)\right] H_{1}
\end{array} .\right.
\end{aligned}
$$


Based on the distributions above, detection accuracy of $S U_{1}$ and $S U_{2}$, evaluated in term of false alarm and missed detection probabilities, can be expressed as

$$
\begin{aligned}
& P_{f 1}=Q\left(\frac{\lambda_{1}-m_{10}}{\delta_{10}}\right), \\
& P_{m 1}=1-Q\left(\frac{\lambda_{1}-m_{11}}{\delta_{11}}\right), \\
& P_{f 2}=Q\left(\frac{\lambda_{2}-m_{20}}{\delta_{20}}\right), \\
& P_{m 2}=1-Q\left(\frac{\lambda_{2}-m_{21}}{\delta_{21}}\right),
\end{aligned}
$$

where $Q(\cdot)$ is the $\mathrm{Q}$-function.

\section{Relay policy optimization}

In our scheme, $S U_{2}$ takes $T_{R}=\eta T_{d}$ time off from the entire $T_{d}$ period to act as the relay node and help $S U_{1}$, while its detection time is reduced to $(1-\eta) T_{d}$. If adopting a conservative relay policy and choosing a small $T_{R}$, little help will be offered to $S U_{1}$. On the contrary, if adopting an aggressive relay policy and selecting a large $T_{R}$, $S U_{2}$ itself will suffer from severe performance degradation. This section deals with the issue of how to determine relay policy

$$
\eta \triangleq \frac{T_{R}}{T_{d}},(0<\eta<1)
$$

where the more $\eta$ approaches 0 , the more conservative relay policy is; the more $\eta$ approaches 1 , the more aggressive relay policy is.

Many criteria can be implemented to find an optimal $\eta$, and this paper gives two examples of balancing detection accuracy and maximizing detection agility.

\subsection{Balancing detection accuracy}

Under the criterion of balancing detection accuracy, our aim is to let different SUs achieve the same detection accuracy to avoid either of them producing too many detection errors. Without loss of generality, consider the energy detector with constant false alarm probability $\alpha$. Then, the same detection accuracy of $S U_{1}$ and $S U_{2}$ can be expressed as

$$
\left\{\begin{array}{l}
P_{f 1}=P_{f 2}=\alpha \\
P_{m 1}=P_{m 2}
\end{array}\right.
$$

Substituting (17), (18), (19) and (20) into (22) and eliminating $\lambda_{1}$ and $\lambda_{2}$, an implicit equation for $\eta$ is obtained

$$
\begin{aligned}
& Q^{-1}(\alpha) \sqrt{2}-\sqrt{n(1-\eta)} \gamma_{2} \\
& =Q^{-1}(\alpha) \sqrt{2} \sqrt{\frac{1+6 \eta}{\phi^{2}+\eta(4+2 \phi)}}-\frac{\sqrt{n}\left(\gamma_{1}+\eta \gamma_{2}\right)}{\sqrt{\phi^{2}+\eta(4+2 \phi)}},
\end{aligned}
$$

where $\phi \triangleq\left(1+\gamma_{1}\right) /\left(1+\gamma_{2}\right)$. According to (23), the desired relay policy, namely $\eta_{\text {opt }}$, is determined by parameters $\alpha$, $n, \gamma_{1}$, and $\gamma_{2}$.

Considering the low SNR scenarios in which the signal from PU is usually very weak $[7,8,20]$, we have $(1+$ $\left.\gamma_{1}\right) /\left(1+\gamma_{2}\right) \approx 1$. Then, an interesting conclusion can be derived as

$$
\eta_{\text {opt }}(\rho)=\frac{5 \rho-2+\sqrt{53 \rho^{2}-20 \rho-24}}{14 \rho},
$$

where $\rho=\gamma_{2} / \gamma_{1}(\rho>1)$ is the ratio of $\gamma_{2}$ to $\gamma_{1}$. That is, the optimal relay policy $\eta_{\text {opt }}$ to balance the detection accuracy of $S U_{1}$ and $S U_{2}$ merely depends on their SNR gap $\rho$.

Moreover, since $\eta_{\text {opt }}$ is monotonically increasing in $\rho$, its lower and upper bounds are given by

$$
\begin{aligned}
& \lim _{\rho \rightarrow 1^{+}} \eta_{\text {opt }}(\rho)=\frac{3}{7} \approx 42.9 \%, \\
& \lim _{\rho \rightarrow \infty} \eta_{\text {opt }}(\rho)=\frac{5+\sqrt{53}}{14} \approx 87.2 \% .
\end{aligned}
$$

Therefore, $S U_{2}$ should spend at least $42.9 \%$ and at most $87.2 \%$ of its detection period in assisting $S U_{1}$ regardless of $\rho$.

It should be pointed out that, if $S U_{1}$ and $S U_{2}$ have the same $\mathrm{SNR}$, their detection accuracy will be originally equal and no relay is required to balance their accuracy, namely $\eta_{\text {opt }}(1)=0$. This phenomenon is not in contradiction with our results. When deducing (24), a quadratic equation is solved and two solutions are produced,

$$
\eta_{\text {opt }}(\rho)=\frac{5 \rho-2 \pm \sqrt{53 \rho^{2}-20 \rho-24}}{14 \rho} .
$$

Note that another solution $\left(5 \rho-2-\sqrt{53 \rho^{2}-20 \rho-24}\right)$ $/(14 \rho)$ has been discarded as the relay policy cannot be negative. Its right-hand limit is 0 as $\rho$ approaches 1 .

\subsection{Maximizing detection agility}

The concept of agility is developed to describe the ability of SUs to quickly vacate the licensed spectrum band after PU emerges [13, 19], and it can be evaluated in terms of the time needed to vacate the band, namely the vacating time,

$$
T_{\eta}=k_{\eta}\left(T_{d}+T_{c}\right),
$$

where $k_{\eta}$ denotes the number of detections required to vacate the band. Obviously, larger $T_{\eta}$ results in lower agility, and smaller $T_{\eta}$ leads to higher agility.

Detailedly, in the two-SU CR network of this paper, since $S U_{1}$ and $S U_{2}$ usually share the licensed band by using different code or frequency sub-channels when PU is absent, the band is vacated only if they both stop transmitting. Moreover, as there exists no fusion center in the CR network, if one SU finds the presence of PU out, it can hardly inform another $\mathrm{SU}$ about this message and ask it to vacate 
the band as considered in [13]. Consequently, the licensed spectrum band can be completely vacated only in case that both $S U_{1}$ and $S U_{2}$ have detected out the presence of PU separately.

Note that, due to the inevitable missed detection errors, how many detections are required by $S U_{1}$ and $S U_{2}$ to both detect out the presence of PU after PU emerges is not fixed. This number $k_{\eta}$ is essentially a random variable, and the probability of $k_{\eta}=l$ is given by

$$
\begin{aligned}
\operatorname{Pr}\left(k_{\eta}=l\right)= & P_{m 1}^{l-1}\left(1-P_{m 1}\right) \cdot\left(1-P_{m 2}^{l}\right) \\
& +\left(1-P_{m 1}^{l}\right) \cdot P_{m 2}^{l-1}\left(1-P_{m 2}\right) \\
& -P_{m 1}^{l-1}\left(1-P_{m 1}\right) P_{m 2}^{l-1}\left(1-P_{m 2}\right) .
\end{aligned}
$$

Based on (29), the mean of $k_{\eta}$ can be expressed as

$$
\begin{aligned}
E\left\{k_{\eta}\right\} & =\sum_{l=1}^{\infty} l \cdot \operatorname{Pr}\left(k_{\eta}=l\right) \\
& =\frac{1}{1-P_{m 1}}+\frac{1}{1-P_{m 2}}-\frac{1}{1-P_{m 1} P_{m 2}} .
\end{aligned}
$$

Consequently, the average vacating time in this paper yields to

$$
\begin{aligned}
E\left\{T_{\eta}\right\} & =E\left\{k_{\eta}\left(T_{d}+T_{c}\right)\right\} \\
& =\left(\frac{1}{1-P_{m 1}}+\frac{1}{1-P_{m 2}}-\frac{1}{1-P_{m 1} P_{m 2}}\right)\left(T_{d}+T_{c}\right) .
\end{aligned}
$$

Given a specific relay policy $\eta$, both $P_{m 1}$ and $P_{m 2}$ can be calculated according to (18) and (20). Substituting these missed detection probabilities into (31), the average vacating time $E\left\{T_{\eta}\right\}$ can also be obtained theoretically. Repeating two steps above and implementing numerical search, it is easy to find out the optimal relay policy $\eta_{\text {opt }}$, with which $E\left\{T_{\eta}\right\}$ can be minimized. In partial scenarios, if $S U_{2}$ spends $\eta_{\text {opt }}$ portion of its detection period acting as the relay node, detection agility of the entire CR network can be maximized eventually.

Furthermore, in order to highlight the benefit of partial relay-based cooperation, this paper compares its detection agility with that of non-cooperation and defines the agility gain as follows,

$$
\tau_{\eta} \triangleq \frac{E\left\{T_{0}\right\}}{E\left\{T_{\eta}\right\}}
$$

where $E\left\{T_{0}\right\}$ is the average vacating time of noncooperation. The detailed analysis on agility gain can be found in the next section.

\section{Simulation results}

This section provides some simulation results to illustrate the partial relay-based cooperative PU detection and its relay policy. The simulation settings are $\alpha=0.1, \gamma_{1}=$
$-20 d B, n=600, T_{d}=T_{c}=n f_{s}$, and $f_{s}=21.524476 \mathrm{M}$ samples/sec [21]. Unless otherwise specified, we assume $\kappa \cdot \gamma_{h}=1$.

Figure 3 plots the missed detection probabilities of $S U_{1}$ and $S U_{2}$ versus the relay policy under different SNRs of $\gamma_{2}=-8,-10,-12,-14 \mathrm{~dB}$. Obviously, in case of noncooperation $(\eta=0), S U_{1}$ causes too many detection errors as its SNR is extremely low. So no matter how well $S U_{2}$ performs, $\mathrm{PU}$ is interfered seriously. As $\eta$ increases, $P_{m 1}$ decreases and $P_{m 2}$ increases; the larger $\gamma_{2}$ is, the more sharply $P_{m 1}$ and $P_{m 2}$ change. It is because $S U_{2}$ sacrifices $\eta T_{d}$ time to aid $S U_{1}$ by relay, and more powerful $S U_{2}$ could offer more help. The curves of $P_{m 1}$ and $P_{m 2}$ intersect eventually. This indicates the detection accuracy of two SUs has been balanced. Note that the points of $\left(\eta_{o p t}, P_{m}\right)$ calculated according to (24) and (18) and the bounds of $\eta_{\text {opt }}$ derived from (25) and (26) are also plotted in this figure. Since all curve intersections agree well with these points and bounds, theoretical analysis on relay policy is verified. If $\eta$ continues increasing and becomes larger than $\eta_{\text {opt }}$, the performance of $S U_{1}$ will get better while $S U_{2}$ will suffer from excessive missed detection errors. That is not what we expected either.

According to (24), the optimal relay policy for balancing detection accuracy merely depends on the SNR ratio of $S U_{2}$ to $S U_{1}$. Figure 4 depicts their relationship. As shown in this figure, $S U_{2}$ should spend at least $42.9 \%$ of its detection period on relay. When the SNR ratio $\rho$ increases, the optimal relay policy $\eta_{\text {opt }}$ grows, but its growth speed gradually slows down. If $\rho$ is larger than $15 \mathrm{~dB}$, increase in $\rho$ causes little impact on $\eta_{\text {opt }}$, and $\eta_{\text {opt }}$ approaches $82.7 \%$. That is, $S U_{2}$ should spend at most $82.7 \%$ of its detection period acting as the relay.

Figure 5 plots the curves of average vacating time $E\left\{T_{\eta}\right\}$ versus relay policy $\eta$ for different SNRs of $S U_{2}\left(\gamma_{2}=\right.$

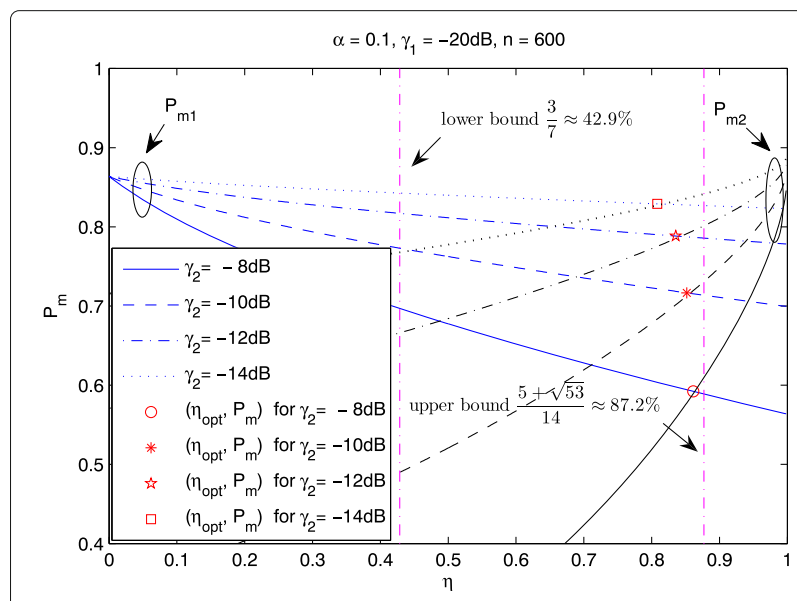

Fig. 3 Missed detection probabilities of $S U_{1}$ and $S U_{2}$ versus relay policy under different SNRs of $\mathrm{SU}_{2}$ 


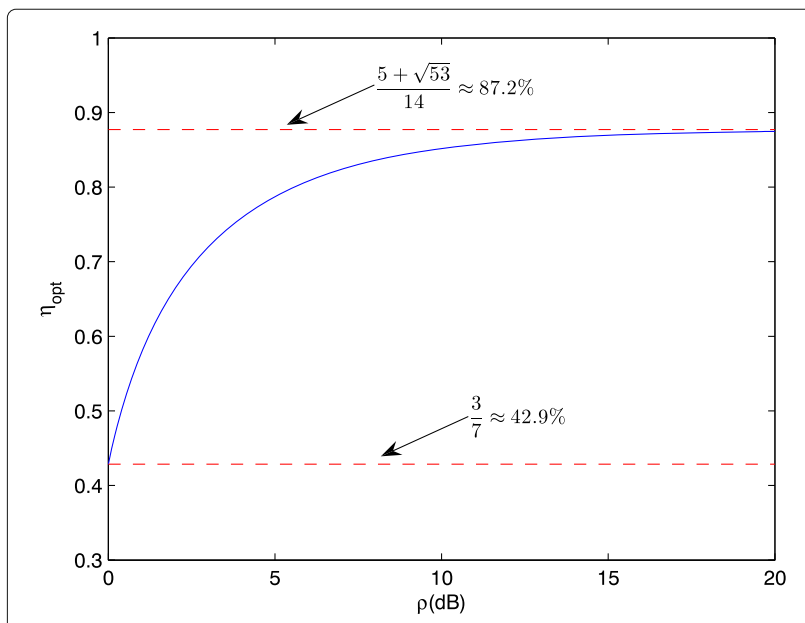

Fig. 4 Optimal relay policy versus SNR ratio under balancing detection accuracy criterion

$0,-5,-10,-15 \mathrm{~dB})$. In this figure, all curves are concave, which means that both excessively conservative $\eta$ and excessively aggressive $\eta$ result in high vacating time and low agility. Therefore, it is necessary to choose an appropriate $\eta$. Implementing numerical search as suggested in Section 4, we derive the optimal relay policy $\eta_{\text {opt }}$ for each $\gamma_{2}$ and plot them with red diamond markers. As shown in the figure, all markers are located at the bottom of the corresponding vacating time curves, so our method of deducing $\eta_{\text {opt }}$ is verified. In addition, $\eta_{\text {opt }}$ increases as $\gamma_{2}$ increases. This phenomenon indicates that $S U_{2}$ should adopt a more aggressive relay policy if its SNR is higher.

Figure 6 depicts the curves of detection agility gain $\tau_{\eta}$ versus relay policy $\eta$ under different SNRs of $S U_{2}$ $\left(\gamma_{2}=0,-5,-10,-15 \mathrm{~dB}\right)$. The red diamond marker

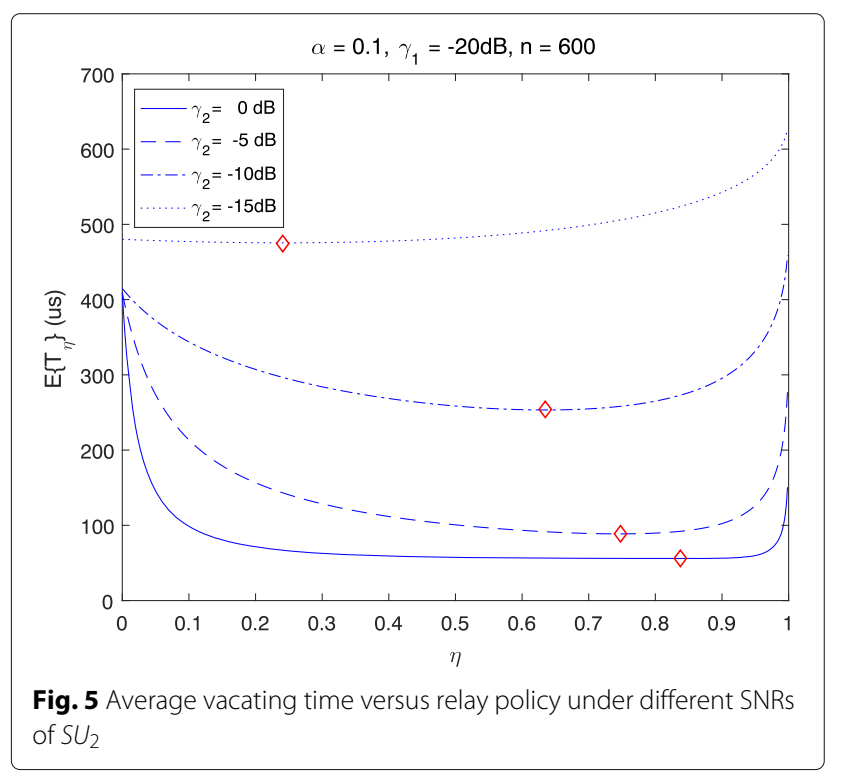

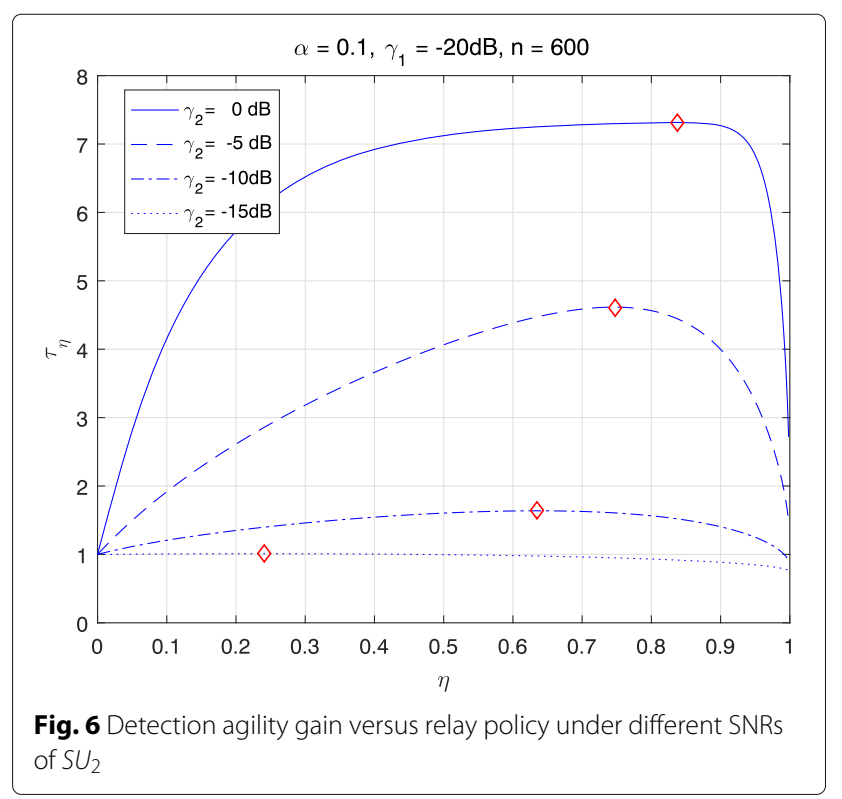

on each curve shows the optimal relay policy $\eta_{\text {opt }}$ that has been discussed in Fig. 5 and its corresponding maximum agility gain. As shown in Fig. 6, all curves approach 1 as $\eta$ approaches 0 . This is because when $\eta$ is very small, $S U_{2}$ spends little time acting as the relay node, and thus produces little agility improvement. Moreover, when $\eta<$ $\eta_{\text {opt }}, \tau_{\eta}$ increases as $\eta$ increases, and its increase speed is higher if $\gamma_{2}$ is larger. For $\gamma_{2}=0 \mathrm{~dB}, \tau_{\eta}$ can reach as much as 7.3. In other words, the detection agility can be improved by $630 \%$. It should also be pointed out that, when $\eta>\eta_{\text {opt }}$, increase in $\eta$ does not cause positive impact on $\tau_{\eta}$ any longer. Especially, when $\gamma_{2}=-15 \mathrm{~dB}$ and $\eta>0.5, \tau_{\eta}$ is smaller than 1 , which indicates that the detection agility turns worse after partial relay. As a result, it is better to avoid adopting an extremely aggressive relay policy when the SNR of $S U_{2}$ is very low.

Finally, in order to investigate the impacts of relay gain $\kappa$ and relay channel $\gamma_{h}$, Fig. 7 considers different values of $\kappa \cdot \gamma_{h}\left(\kappa \cdot \gamma_{h}=6,3,0,-3,-6 \mathrm{~dB}\right)$ with $\gamma_{2}=-5 \mathrm{~dB}$. Obviously, given the same relay policy $\eta$, higher detection agility gain is achieved if $\kappa \cdot \gamma_{h}$ is larger. This phenomenon can be explained as follows. A large $\kappa$ incurs great amplification of relay signal and a high $\gamma_{h}$ (low path loss of relay channel) represents little attenuation in relay signal, both of which are beneficial for our partial relay mechanism. In addition, the highest points of agility gain curves are also marked with red diamond markers in Fig. 7. These markers show that the optimal relay policy should be more conservative (smaller) if $\kappa \cdot \gamma_{h}$ is larger.

\section{Conclusions}

A partial relay-based cooperative PU detection scheme that did not require any dedicate reporting channels was 


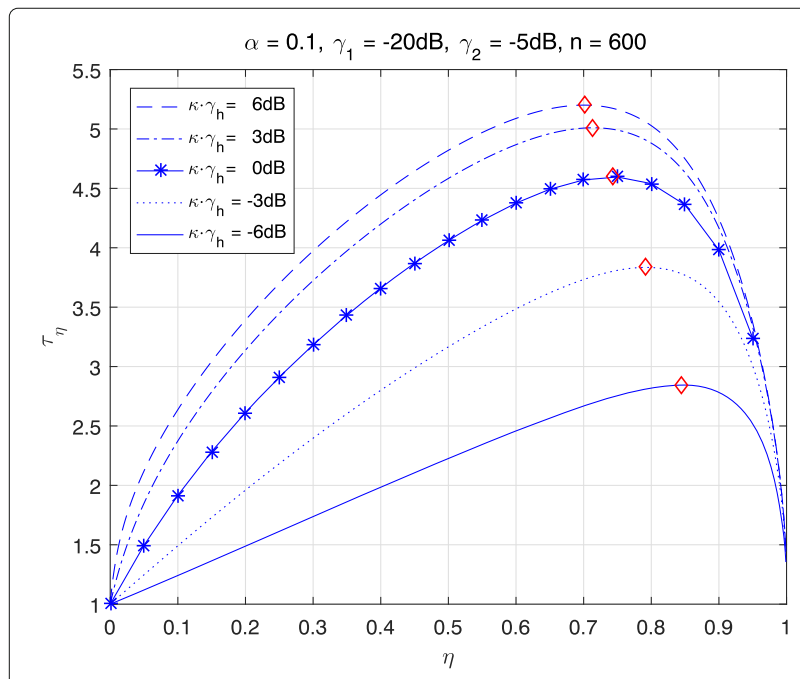

Fig. 7 Impacts of relay gain and relay channel

proposed in this paper. False alarm and missed detection probabilities of the proposed scheme were analyzed. How to determine its relay policy was also investigated. Taking the criterion of balancing detection accuracy for example, closed-form expression, lower bound, and upper bound of the optimal relay policy were deduced. We proved that, in order to avoid either of SUs causing too much interference, the SU with higher SNR should spend at least $42.9 \%$ and at most $87.2 \%$ of its detection period acting as the relay node. Considering the criterion of maximizing detection agility, we showed that, although the performance of relaying SU was inevitably impaired, detection agility of the whole CR network could be improved by as much as $630 \%$ via choosing an optimal relay policy.

\section{Acknowledgements}

The authors would like to thank the National Natural Science Foundation of China (grant nos. 61201264 and 61362018), China Scholarship Council (grant no. 201508350023), and Huaqiao University (grant no.13BS101) for their supports.

\section{Authors' contributions}

The majority of this work is done by SP. All other authors revised the manuscript. All authors read and approved the final manuscript.

\section{Competing interests}

The authors declare that they have no competing interests.

\section{Publisher's Note}

Springer Nature remains neutral with regard to jurisdictional claims in published maps and institutional affiliations.

\footnotetext{
Author details

${ }^{1}$ College of Information Science and Engineering, Huagiao University, 668 Jimei Avenue, 361021 Xiamen, China. ${ }^{2}$ Department of Electrical and Computer Engineering, Stevens Institute of Technology, Castle Point, Hoboken, 07030 USA. ${ }^{3}$ National Mobile Communications Research Laboratory, Southeast University, 2 Sipailou, 210096 Nanjing, China.
}

\section{References}

1. H Zhu, J Wang, Chunk-based resource allocation in OFDMA systems-part I: chunk allocation. IEEE Trans. Commun. 57(9), 2734-2744 (2009)

2. H Zhu, J Wang, Chunk-based resource allocation in OFDMA systems-part II: joint chunk, power and bit allocation. IEEE Trans. Commun. 60(2), 499-509 (2012)

3. H Zhu, Radio resource allocation for OFDMA systems in high speed environments. IEEE J. Sel. Areas Commum. 30(4), 748-759 (2012)

4. J Mitola, GQ Maguire, Cognitive radio: making software radios more personal. IEEE Pers. Commun. 6(4), 13-18 (1999)

5. Q Zhao, BM Sadler, A survey of dynamic spectrum access. IEEE Signal Process. Mag. 24(3), 79-89 (2007)

6. C-S Sum, GP Villardi, MA Rahman, T Baykas, HN Tran, Z Lan, C Sun, Y Alemseged, J Wang, C Song, C-W Pyo, S Filin, H Harada, Cognitive communication in TV white spaces: an overview of regulations, standards, and technology. IEEE Commun. Mag. 51(7), 138-145 (2013)

7. J Unnikrishnan, W Veeravalli, Cooperative sensing for primary detection in cognitive radio. IEEE J. Sel. Topics Signal Process. 2(1), 18-27 (2008)

8. IF Akyildiz, BF Lo, R Balakrishnan, Cooperative spectrum sensing in cognitive radio networks: a survey. Phys. Commun. 4, 40-62 (2011)

9. J Ma, Y Li, in 2007 IEEE Global Telecommunications Conference (GLOBECOM). Soft combination and detection for cooperative spectrum sensing in cognitive radio networks (IEEE, Washington, DC, 2007), pp. 3139-3143

10. W Choi, M-G Song, J Ahn, G-H Im, Soft combining for cooperative spectrum sensing over fast-fading channels. IEEE Commun. Lett. 18(2), 193-196 (2014)

11. W Han, J Li, Z Li, J Si, Y Zhang, Efficient soft decision fusion rule in cooperative spectrum sensing. IEEE Trans. Signal Process. 61(8), 1931-1943 (2013)

12. Z Li, FR Yu, M Huang, A distributed consensus-based cooperative spectrum-sensing scheme in cognitive radios. IEEE Trans. Veh. Technol. 59(1), 383-393 (2010)

13. G Ganesan, Y Li, Cooperative spectrum sensing in cognitive radio, part i: two user networks. IEEE Trans. Wirel. Commun. 6(6), 2204-2213 (2007)

14. J Si, Z Li, J Chen, H Huang, in 2012 IEEE International Conference on Communications (ICC). On the performance of cognitive relay networks with cooperative spectrum sensing (IEEE, Ottawa, 2012), pp. 1709-1714

15. Y Zou, Y-D Yao, B Zheng, Cooperative relay techniques for cognitive radio systems: spectrum sensing and secondary user transmissions. IEEE Commun. Mag. 50(4), 98-103 (2012)

16. Y Zou, Y-D Yao, B Zheng, A selective-relay based cooperative spectrum sensing scheme without dedicated reporting channels in cognitive radio networks. IEEE Trans. Wirel. Commun. 10(4), 1188-1198 (2011)

17. I Krikidis, J Thompson, J Thompson, N Goertz, Amplify-and-forward with partial relay selection. IEEE Commun. Lett. 12(4), 235-237 (2008)

18. S Peng, S Shu, X Yang, X Cao, in 2012 IEEE Wireless Communications and Networking Conference (WCNC). Relay based cooperative spectrum sensing in distributed cognitive radio networks (IEEE, Paris, 2012), pp. 1370-1374

19. G Ganesan, $Y L i$, Cooperative spectrum sensing in cognitive radio, part ii: multiuser networks. IEEE Trans. Wirel. Commun. 6(6), 2214-2222 (2007)

20. Y-C Liang, Y Zeng, ECY Peh, AT Hoang, Sensing-throughput tradeoff for cognitive radio networks. IEEE Trans. Wirel. Commun. 7(4), 1326-1337 (2008)

21. Y Zeng, Y-C Liang, in 2007 2nd IEEE International Symposium on New Frontiers in Dynamic Spectrum Access Networks (DySPAN). Covariance based signal detections for cognitive radio (IEEE, Dublin, 2007), pp. 202-207 